\title{
Little auks under the midnight sun: diel activity rhythm of a small diving seabird during the Arctic summer
}

\author{
Katarzyna Wojczulanis-Jakubas (1) ${ }^{1}$, Piotr Wąż² \& Dariusz Jakubas (1) \\ 'Department of Vertebrate Ecology and Zoology, Faculty of Biology, University of Gdańsk, Gdańsk, Poland; \\ ${ }^{2}$ Department of Nuclear Medicine, Medical University of Gdanńsk, Gdańsk, Poland
}

\begin{abstract}
Many animal species exhibit a diel, 24-hr pattern of activity, which is steered by timing cues, with the daily light-dark cycle considered the most powerful. This cue, however, is reduced in polar zones under continuous daylight conditions associated with the midnight sun. The rhythm of animal behaviour under such conditions is poorly understood. Here, we examine periodicity and patterns of daily activity (colony attendance and foraging) in a High-Arctic seabird, the little auk (Alle alle). We demonstrated a regular rhythm of colony attendance at the population level, with birds being the most abundant in the colony during hours of relatively low sun elevation. This pattern is likely to be associated with predation pressure that may be perceived by birds as lower during hours with low sun elevation, because of better predator detectability. Regarding rhythms at an individual level, however, we found the most common periodicity to be $23.2 \mathrm{hr}$ (range from $19.9 \mathrm{hr}$ to $30.8 \mathrm{hr}$ ) but no clear pattern of daily colony attendance of individuals. Such a flexibility in daily rhythms indicates that individuals may become arrhythmic in regard to the 24-hr environmental cycle, despite regularities observed at the population level. Finally, we compared males and females in terms of daily activity patterns but we did not find significant sex differences.
\end{abstract}

\section{Keywords}

circadian rhythm; Alle alle; polar day; Zeitgeber; 24-hour daylight; dovekie

\section{Correspondence}

Katarzyna Wojczulanis-Jakubas, Department of Vertebrate Ecology and Zoology, Faculty of Biology, University of Gdańsk, Wita Stwosza 59, PL-80-308 Gdańsk, Poland. E-mail: biokwj@univ.gda.pl

\section{Abbreviations \\ GAM: generalized additive model}

To access the supplementary material, please visit the article landing page

\section{Introduction}

Many animal species exhibit a diel pattern of activity, which is steered by external (daily light-dark cycle and prey abundance) and endogenous timing cues (e.g., physiological condition, as reviewed in Steiger et al. [2013]). Of those, the daily light-dark cycle is considered the most powerful cue to which most animals are synchronized. However, in polar regions, the strength of this cue is limited during continuous summer daylight (Steiger et al. 2013). Behavioural and physiological responses of polar vertebrates to such conditions are diverse (van Oort et al. 2000; Williams et al. 2015). They may react to conditions associated with the midnight sun by: (1) becoming arrhythmic, (2) entraining to weaker light cues (e.g., light intensity, polarization or sun azimuth diel changes) or (3) relying on endogenous rhythms, that is, "free-running" with respect to the 24-hr cycle (Steiger et al. 2013; Ashley et al. 2014). Recent studies on free-living, Arctic breeding birds have revealed diversity and flexibility in daily activity rhythms, with species-, sex- and/or breeding stage-specific strategies (Steiger et al. 2013; Ashley et al. 2014; Bulla et al. 2016). A comparative analysis would allow for the investigation of the relationships between daily activity patterns, breeding stages and life-history traits. However, before final conclusions about the mechanisms and phylogenetic origin of the observed patterns can be generated, more data on species breeding in polar regions should be collected.

In this study, we examined patterns of daily activity in the little auk (Alle alle), also known as the dovekie (Fig. 1), a small zooplanktivorous and colonially breeding seabird. The species breeds exclusively in the High Arctic, utilizing rock crevices in mountain slopes. It is considered the most abundant alcid in the Palearctic (Stempniewicz 2001) and as such represents a perfect model species to study circadian rhythms in polar vertebrates.

Although the pattern of little auk colony attendance has been examined at the local population scale (Stempniewicz 1986), the daily activity rhythm of individuals 


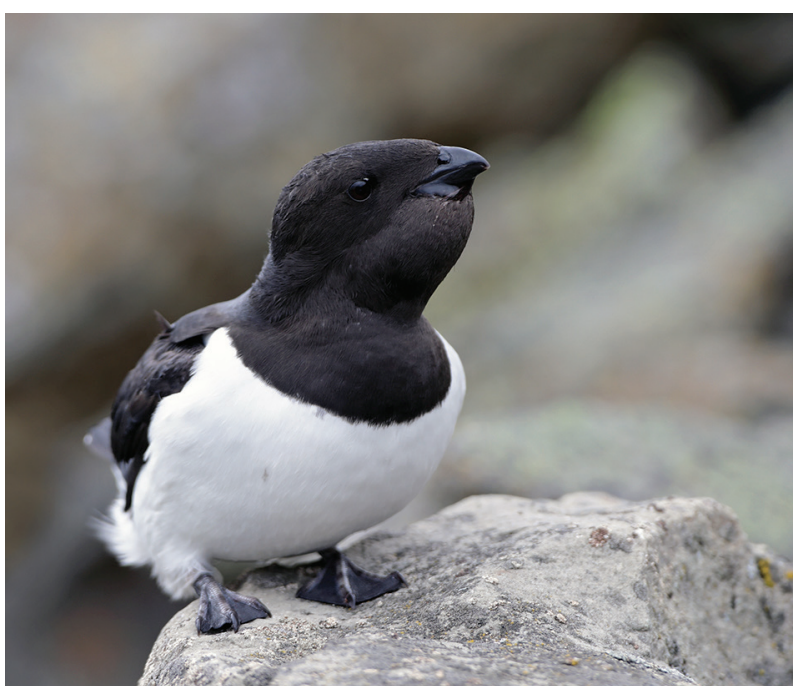

Fig. 1 Adult little auk with full gular pouch, carrying food for its chick. (Photo credit: Piotr Jończyk.)

has not yet been systematically examined. Stempniewicz (1986) has reported that birds exhibit various patterns of colony attendance throughout the 24-hr cycle during the chick-rearing period. Nevertheless, the rhythm recorded at colony scale does not necessarily reflect the rhythm of an individual. Further, variability in foraging trip duration (ranging from $1.3 \mathrm{hr}$ to $22.7 \mathrm{hr}$; [Welcker, Harding et al. 2009; Jakubas et al. 2014]) and necessity to adjust chick provisioning to the breeding partner (Wojczulanis-Jakubas et al. 2018) can potentially compromise 24-hr periodicity of particular individuals (Favreau et al. 2009).

Here we examined birds' activity patterns and periodicity at population and individual levels. As recent studies have shown that daily patterns can be sex-specific (e.g., Steiger et al. 2013; Bulla et al. 2016; Huffeldt \& Merkel 2016), we considered males and females separately. We focused on the chick-rearing period for the studied population (July/August), as Stempniewicz did (1986). In contrast to the incubation period, during chick rearing it is not a requirement that one of the parent birds stay on the nest. Chick rearing is therefore the period during which individual choice regarding presence in the colony plays a much stronger role than during incubation (Pietz 1986; Bulla et al. 2014; Bulla et al. 2016).

\section{Materials and methods}

\section{Field methods}

We carried out the study in a little auk colony in Magdalenefjorden, north-west Spitsbergen $\left(79^{\circ} 35 \mathrm{~N}, 11^{\circ} 05 \mathrm{E}\right)$, Svalbard. The study site is one of the largest breeding aggregations of this species on Spitsbergen (Isaksen 1995; Keslinka et al. 2019). We collected data during five 48-hr continuous observations: two in 2009 (during the second and third week of the chick-rearing period, determined by hatching dates in the group of followed nests) and three in 2010 (second, third and fourth week of the chick-rearing period; see details in Table 1). To establish a daily distribution of the birds' colony attendance at the local population scale, we counted all the birds present in the colony patch (area of ca. $1200 \mathrm{~m}^{2}$ ) every 10-30 min during each observation. We counted birds at the moments when they had not been disturbed by predators for at least 5 min, which minimized an error of the estimated number. Birds present in the colony are frequently scared away by predators, which appear up to 16 times per hour (Wojczulanis-Jakubas et al. 2005). After getting scared and flying away, the birds may land on a neighbouring colony patch, but within a few minutes they move to their own patch (personal observations). Owing to the little auk nesting preferences-crevices in rock boulders of a given size (Keslinka et al. 2019)—and topography of the colony area, boundaries of each colony patch are easily distinguished: patches are separated by ground areas of different structure (size and density of rocks) compared to those in colony patches. The studied patch was chosen because it offered a suitable observation spot located ca. $20 \mathrm{~m}$ away, a distance that minimized disturbance but was close enough to allow observers to identify individually marked birds. On the basis of visual comparisons of all the colony patches in the field, we considered the study patch as representative for the local population (Supplementary Fig. S1). The exact number of nests on the studied patch was not known as it is difficult to estimate the population of a crevice-nesting species, but we estimated the density of nests at 1.6 pairs per $1 \mathrm{~m}^{2}$, following methods described by Keslinka et al. (2019). Owing to the long lifespan and high site fidelity of little auks (Stempniewicz 2001), the number of nests on the patch was expected to be stable over the two consecutive years of the study.

To examine the individual daily activity patterns and periodicity, we followed individually marked birds during the five periods of observations. For marking, we captured the birds at the end of the incubation period or during the early chick-rearing period (ca. two weeks before the first observation in a given season). We captured adults by hand at the nest while they were incubating eggs. We marked the birds with a combination of individually specific colour leg-rings (three plastic and one metal; two rings on each leg) and dyes applied to the breast feathers. This double marking system allowed us to quickly and reliably identify an individual when it appeared on the colony patch. Colour marks on breast feathers were visible from a distance even without binoculars; when there was 
any doubt, looking at the unique combination of colour rings read through binoculars helped to identify the individual. We were able to follow all marked individuals because all the focal nests were located relatively close to each other (within the study colony patch), and there was never a time when all the marked individuals were on the patch simultaneously. Pairs of observers (changing every 4-8 hr) watched the colony patch continuously and noted the presence of marked birds every $10 \mathrm{~min}$. Information about gular pouch fullness (indicating food being delivered to the chick) was also recorded. Noting individual presence ( 1 ) and absence (0) every $10 \mathrm{~min}$, we obtained a total of 288 data points for each individual during each observation session. We considered a bird's absences from the colony (sequence of zeros in the time series) that were followed by reappearance with a full gular pouch as foraging trips. We captured and observed in total 53 individuals, with 25 being observed in both seasons. The number of birds observed during the season varied slightly between the observations (Table 1) on account of predation, late marking or early completion of breeding.

We monitored focal nests to establish the hatching date, inspecting them daily for a week before hatching. The majority of chicks (75\%, $N=20$; each season) hatched within 6 days. Owing to this synchrony of breeding and quite consistent timing of breeding over two seasons (Moe et al. 2009), we knew when to expect the first hatchlings and could assume a similar age for all chicks in the studied colony patch (Table 1).

\section{Data analysis}

To examine the daily pattern of colony attendance of the little auk at the population level, we first plotted the number of birds present in the colony plot per hour, separately for particular observations (Supplementary Fig. S2). To understand the mechanism driving the observed pattern, we analysed the number of birds at the colony patch (response variable) in relation to sun elevation (explanatory variable), using a GAM, with Gaussian family for the error distribution. We obtained the sun elevation data

Table 1 Characteristics of the observations performed in the little auk colony.

\begin{tabular}{lccc}
\hline Season & $\begin{array}{c}\text { Onset of the observation } \\
\text { (date and hour) }\end{array}$ & $\begin{array}{c}\text { Average chicks } \\
\text { age (d) }\end{array}$ & $\begin{array}{c}\text { Number of } \\
\text { pairs }\end{array}$ \\
\hline 2009 & 27 July, 07:30 & 10 & 18 \\
2009 & 04 August, 13:00 & 18 & 16 \\
2010 & 23 July, 07:40 & 11 & 19 \\
2010 & 01 August, 07:30 & 20 & 20 \\
2010 & 06 August, 20:00 & 24 & 8 \\
\hline
\end{tabular}

for the study site from https://www.sunearthtools.com/ dp/tools/pos_sun.php?lang=en (access date: 21 February 2019). Analysing the sun elevation data, we considered each observation separately. However, given the similarity in the patterns of sun elevation and colony attendance across the observation sessions (Supplementary Fig. S2), we pooled data of all the observations and averaged both the sun elevation and the number of birds in the colony patch per hour to illustrate the GAM results (Fig. 2).

Considering presence/absence data of marked individuals, we usually treated individuals and observations separately as many birds were repeatedly observed across the study period (implying a possible issue of pseudoreplication). Firstly, for each individual/observation, we plotted an actogram showing presence/absence in the colony (e.g., Fig. 3, Supplementary Fig. S3a-d). Secondly, for each individual/observation, we plotted autocorrelation correlograms (e.g., Fig. 4, Supplementary Fig. S4) to examine their periodicity in the colony attendance. For that purpose, we established the birds presence/absence $(1 / 0)$ status for each observation hour ( 1 if bird was seen at least once in a given hour, 0 when it was not observed during an hour) and used a lag time of 1 hr for the autocorrelation analysis. Although the resulting correlograms revealed some periodicity, the patterns were not clear for all individuals (Supplementary Fig. S4). To identify important periods of individuals' presence/absence in the colony, we applied discrete Fourier transform analysis for the established time series. This analysis converts a finite sequence of signal samples $(0 / 1$ sequence for presence/absence in the colony) to a harmonic sequence, and the coefficients can be interpreted as amplitudes of the corresponding harmonic components. The whole spectrum of the harmonic components are frequencies that are odd-integer multiples of the fundamental frequency, with amplitudes decreasing with an increase of harmonic frequencies (Terras 1999). We ran the analysis and presented the results for the pooled data (all individuals and observations combined) as we aimed to establish a general pattern of little auk periodicity in colony attendance. We considered the sexes separately (birds sexed molecularly [Wojczulanis-Jakubas et al. 2006]) in this analysis because of possible sex-specific patterns. To address possible pseudoreplication in the pooled data, we also ran the analysis for each observation (and sex) separately. From each set of results, we selected five frequencies with the highest amplitudes and calculated density of frequency distribution.

To analyse inter-annual similarity of colony attendance for particular individuals, we used cross-correlation analysis. For obvious reasons, we performed this analysis only for individuals that were observed in both study seasons $(N=25$; the first observation in the season 

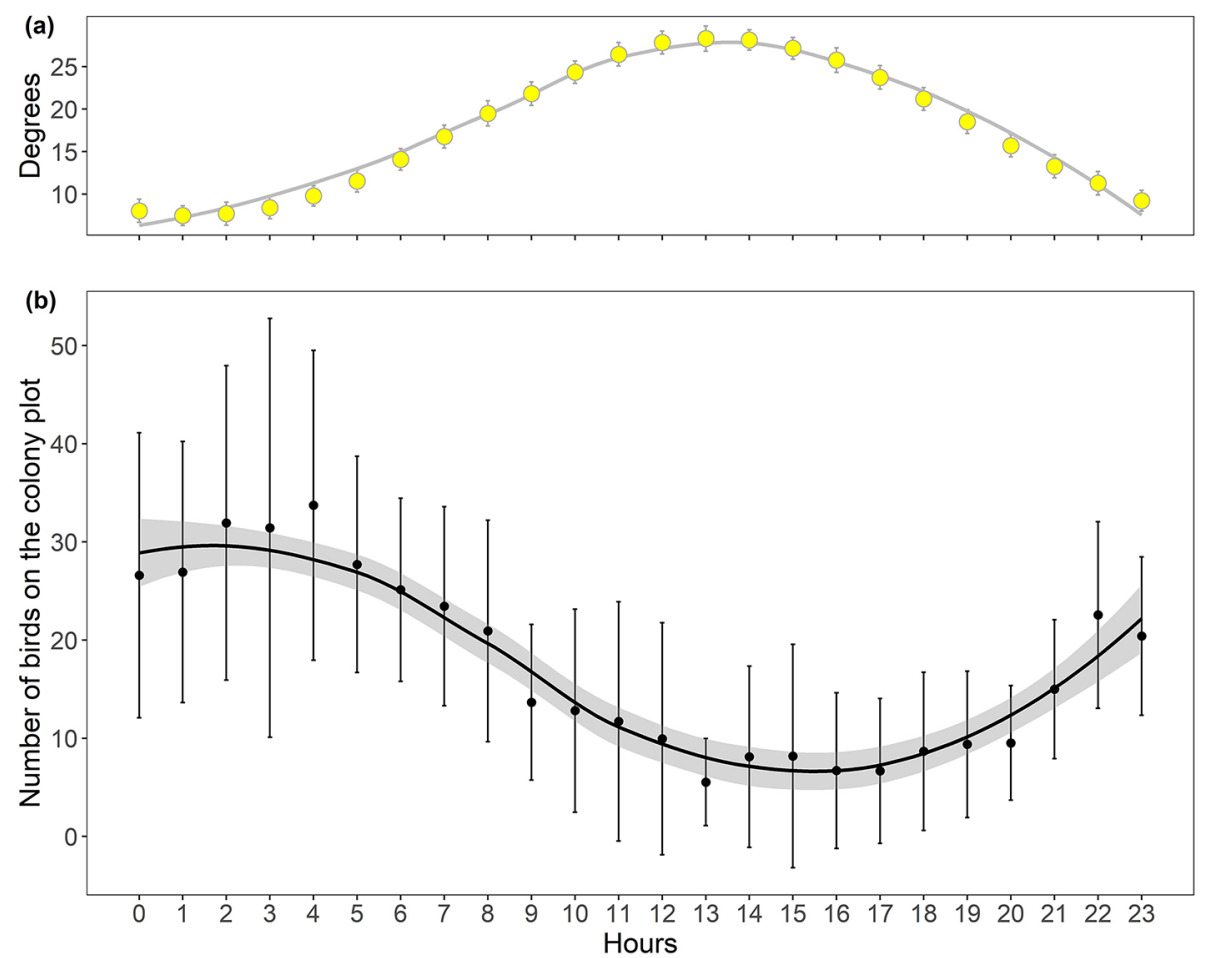

Fig. 2 (a) Daily sun elevation and (b) colony attendance pattern of the little auk. Values are expressed as means (dots) and standard errors (bars), calculated based on five watches performed during the chick-rearing period in the two study seasons. The curves denote smoothed, line patterns for the observed data, with the $95 \%$ confidence interval in (b) shown in grey. The curves were created using the loess method.

considered only, as those started roughly at the same time of day and same day of the chick-rearing period; Table 1). We used magnitude of the cross-correlation coefficient and its location (Lagmax) as an indicator of the phase relationships of the assessed time series, following Huffeldt \& Merkel (2016).

To examine the daily distribution of foraging behaviour, we focused on the arrival of birds at the colony with full gular pouches (Fig. 1). Birds arriving at the colony with full gular pouches were likely to have finished foraging just before their appearance in the colony, and the chick was fed shortly after the parent's arrival at the colony (Jakubas et al. 2014). Given this, by analysing the pattern of chick provisioning, we could elucidate information about the foraging pattern of adults. To analyse this daily distribution of foraging behaviour, we plotted and analysed data separately for the 48-hr observations split into 24-hr intervals (to address possible issues of pseudoreplication), using circular statistics. We also considered males and females separately to examine possible sex differences in the pattern. We assessed daily distribution of foraging (i.e., chick provisioning) using Rayleigh tests. To compare activity rhythm between the sexes, we used Watson's two-sample tests for homogeneity (Pewsey et al. 2014).
We performed all the analyses in the $\mathrm{R}$ environment (version 3.5.2; 2018), using the circular package (Agostinelli \& Lund 2017) to convert hours to circular data and for the Rayleigh test and Watson's two-sample test. For GAM, we used the mgcv package (Wood 2011); and for autocorrelation, cross-correlation and Fourier analyses, we used the functions implemented in basic $\mathrm{R}$.

\section{Ethical note}

The study was not invasive; the only procedure of that nature was handling birds to mark them prior to observations. All the birds were handled with utmost care and released after ca. 5 min of handling without any harm; after release, they were all observed in the colony behaving normally. Fieldwork was performed with permission from the Norwegian Animal Research Committee and the Governor of Svalbard.

\section{Results}

Although the number of observed birds in the studied colony patch varied slightly across the study period (as showed by the difference in position of smooth curves, representing various stages of chick rearing, on the $y$-axis 

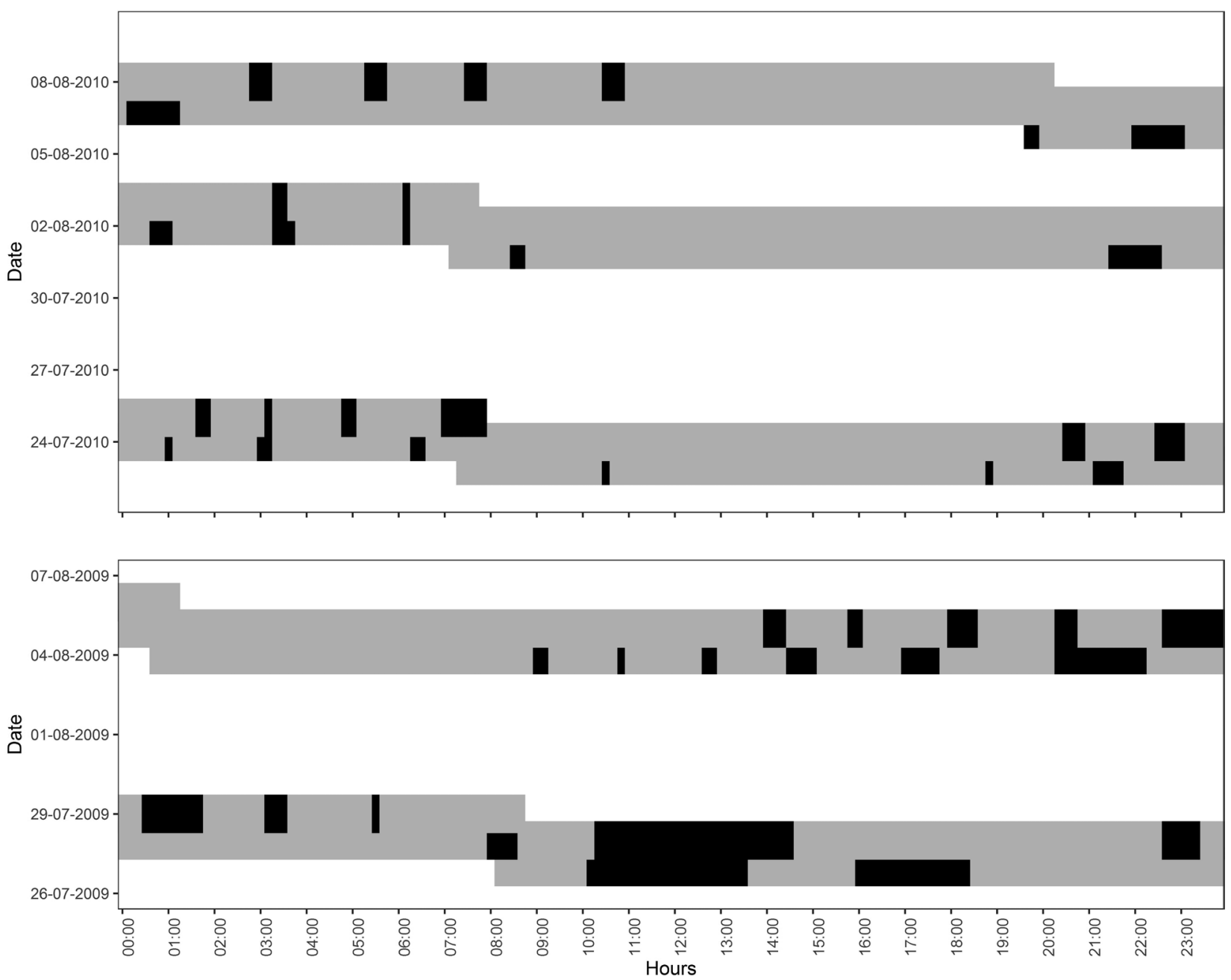

Fig. 3 An example of colony attendance pattern of an individual (ring number 41425), expressed as the bird's presence (black) and absence (grey) in the colony patch, recorded during all the 48-hr observations performed during two (in 2009) and three stages (in 2010) of the chick-rearing period.

of Supplementary Fig. S2), the overall pattern of colony attendance at the colony patch (population level) was similar across the study period (Fig. S2) and reversely reflected sun elevation $(29.5 \%$ of explained deviance, GAM, $F=24.03$, edf $=6.928, p<0.001$; Fig. 2). However, at an individual scale (presence/absence data of individually marked birds), this pattern was not obvious, owing to individuality in colony attendance rhythm, which was not repetitive for all individuals across breeding stages or seasons (e.g., Fig. 3, Supplementary Fig. S3). Autocorrelograms revealed a clear periodicity for some but not all individuals (e.g., Fig. 4, Supplementary Fig. S4). Consistently, the range of cross-correlation coefficient was quite wide, from -0.46 to 0.55 , with Lagmax ranging from -28 to 14 , but it was insignificant for all except one individual (Supplementary Fig. S5). This indicates that birds do attempt to hold a cyclic rhythm but apparently have trouble being consistent. Fourier transform analysis performed on pooled data (all seasons and observations combined) revealed that most of the considered time series were characterized only by several large amplitudes of frequencies. Regardless of the sex, the most common frequency was $23.2 \mathrm{hr}$; few other frequencies of shorter duration also occurred in a noticeable number (Fig. 5). Further, when the Fourier analysis was performed for each season (pooled observations), each stage (pooled seasons) and each sex separately, $23.2 \mathrm{hr}$ was the most common periodicity. The only exceptions were: (a) females during the mid-chick-rearing period (the second observation), with the most common periodicity of $30.8 \mathrm{hr}$; and (b) both sexes during the late chick-rearing period (the third observation), with the most common frequency of $23.6 \mathrm{hr}$ for males, and two frequencies of $19.9 \mathrm{hr}$ and $2.43 \mathrm{hr}$ for females (for all, density $>30$ ). 


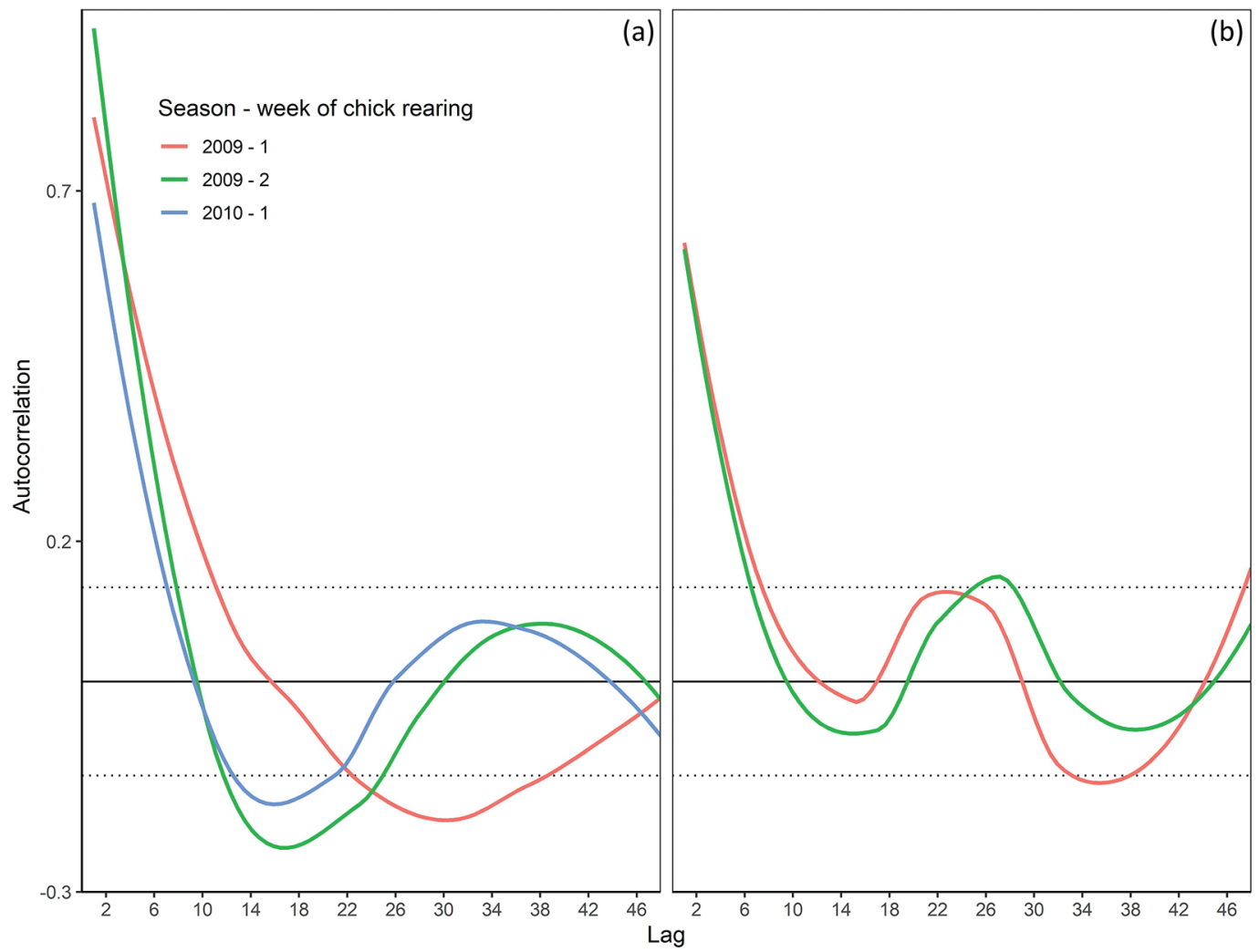

Fig. 4 Example correlograms of two individuals with contrasting patterns-(a) non-consistent and (b) consistent-recorded during the 48-hr observations, performed during two stages of the chick-rearing period and two breeding seasons.
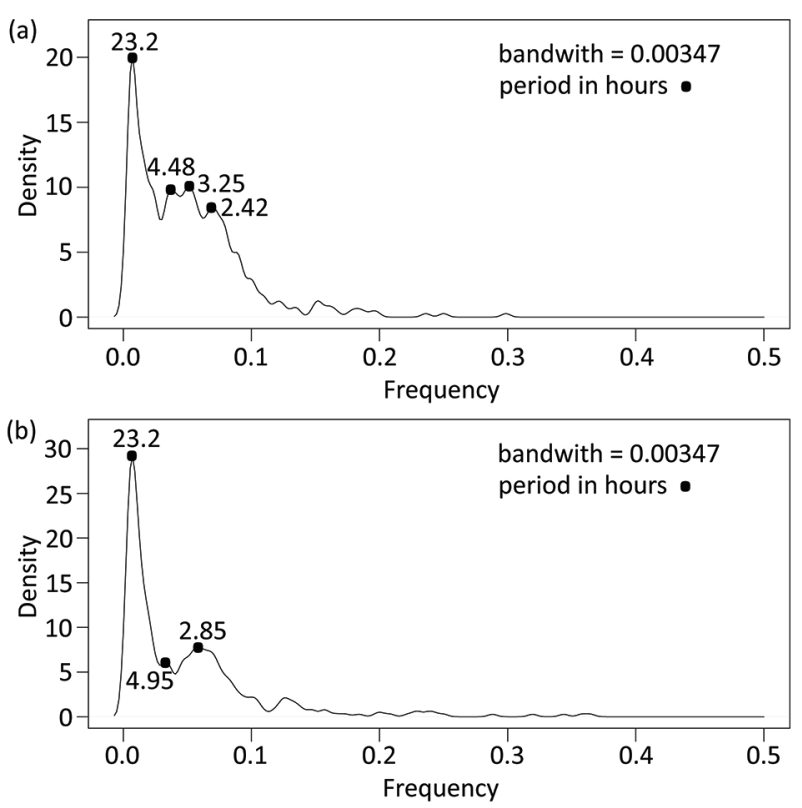

Fig. 5 Density distribution of the most common periods revealed by Fourier analysis, performed on pooled data (all observations and seasons combined), with (a) males ( $n=83$ ) and (b) females ( $n=82$ ) considered separately.
Chicks were provisioned throughout the whole 24-hr cycle, with no noticeable peaks in activity (Rayleigh tests, Supplementary Table S1, Supplementary Fig. S6). The only exception was a single 24-hr interval in one season (labelled 2010_1_l: first part of the early chick-rearing period 2010), when the distribution of daily chick provisioning was not uniform, although that was significant only for females (Supplementary Table S1). Although the mean hour of chick provisioning varied across observation sessions (Supplementary Table S1), there were no apparent patterns that could be biologically explained (Supplementary Fig. S4). Further, no apparent differences between the sexes in the daily distribution of chick provisioning were detected (Watson's two-sample tests: all U2 $<0.176$, with critical value at $p=0.05$, equals 0.187 ; Supplementary Fig. S6).

\section{Discussion}

We demonstrated that little auk colony attendance followed a regular rhythm, with birds being the most abundant during hours of relatively low sun elevation. 
The clear, inverse relationship between birds' colony attendance and sun elevation suggests that even under midnight sun conditions, environmental cues play an important role in shaping the daily rhythm of birds' activity. However, at the individual level, the birds were not able to fully adjust their activity to the environmental cycle. Although $23.2 \mathrm{hr}$ was the most common periodicity, it did not entirely match the 24 -hr cycle.

With the 23.2-hr cycle exhibited over a month, the peak of an individual colony attendance drifted across the entire day. Further, there was little evidence of any strong pattern in colony attendance at the individual level as individuals typically displayed inconsistent daily rhythms. This inconsistency in patterns between the entire colony and individuals may be explained by inter-individual variation in foraging efficiency and energy allocation (e.g., between chick provisioning and self-maintenance). The overall rhythm of the birds' presence in the colony is predictable at the population level (with the highest social activity in the hours of low sun elevation), but at the individual level the pattern is apparently disturbed. Although individuals are most likely to be present in the colony during the hours of low sun elevation, sometimes they are away from the colony during the "night" hours and instead visit the colony at various times during the "day."

Little auk colony attendance during the hours of low sun elevation is consistent with observations from another large colony in south-west Spitsbergen (Hornsund), throughout the same phenological period (Stempniewicz 1986). A possible proximate cause of the observed pattern could be that the low sun position leads to increased "shadowing" over the colony owing to the local topography. Then, the ultimate cause of the observed pattern could be predation pressure, which has been reported to influence animals' diel rhythm (Gliwicz 1986; Hansson et al. 2007). Shadows in the colony might favour little auks to detect the glaucous gull (Larus hyperboreus), a main avian predator of the little auk on Svalbard (Stempniewicz 1995). As field observations show, the light-grey and white plumage of the glaucous gull is fairly conspicuous in low light conditions. Gulls seem to take this into account while hunting: they were observed to camouflage themselves by perching, with their heads lowered, on remnant snow patches in the little auk colony, trying to ambush flying adults (Jakubas \& Wojczulanis-Jakubas 2010). Thus, little auks socializing during relatively low light conditions in the colony may benefit from better predator detection.

Another possible factor influencing animals' daily patterns could be food availability (e.g., Wilson et al. 1989; Hau \& Gwinner 1996; Kumar et al. 2001), as shown in some polar species (van Oort et al. 2007). Little auk colony attendance might inversely mirror zooplankton availability in the species' foraging grounds. However, this argument does not seem to apply for the little auk. Firstly, chick feeding used as a proxy for foraging activity was rather equally distributed throughout the 24-hr cycle. Secondly, diel vertical migration of zooplanktonin the Arctic zone, although evident during day-night cycles, is much less obvious during continuous light conditions (Wallace et al. 2010). Whilst diel migration has been demonstrated in multiple zooplankton species (Błachowiak-Samołyk et al. 2006; Berge et al. 2014), the key prey species utilized by little auk during the chick-rearing period in the study colony (Calanus glacialis; Kwasniewski et al. 2010) remains relatively sedentary in the water column at a depthas much as ca. $40 \mathrm{~m}$ (Błachowiak-Samołyk et al. 2006; Berge et al. 2014), a range regularly exploited by little auks (17-35 m; Falk et al. 2000).

As some studies have demonstrated that daily activity patterns can be sex-specific (Steiger et al. 2013; Bulla et al. 2016), we examined periodicity and daily distribution of chick feeding separately for males and females. Importantly, recent research on another sub-polar and polar alcid, the Brünnich's guillemot (Uria lomvia), has shown inverted colony attendance patterns for the sexes (Huffeldt \& Merkel 2016). Nevertheless, we did not find any pronounced inter-sex differences for the little auk. Overall, both sexes fed the chick equally throughout the day. The sex differences found in the Brünnich's guillemot have been explained in terms of sex-specific variation in foraging strategies (Huffeldt \& Merkel 2016). In the case of little auks, males and females also differ in their foraging strategies, with females spending more time at sea (Welcker, Steen et al. 2009) and delivering more food for the chick in single loads (Wojczulanis-Jakubas et al. 2006). However, these sex-specific differences in little auk foraging do not appear to significantly affect the diel pattern of chick-provisioning behaviour exhibited by the sexes.

Patterns of diel distribution of foraging trips may vary at different stages of the breeding season, such as during the incubation period. All the studies showing sex differences in diel activities of birds have been performed during the incubation or brooding periods (e.g., Steiger et al. 2013; Bulla et al. 2014; Bulla et al. 2016; Huffeldt $\&$ Merkel 2016). At the incubation stage, birds are constrained by incubatory behaviour. However, during chick rearing, the birds are less tied to the nest and are therefore more free regarding their presence/absence in the colony.

We interpreted the most common periodicity $(23.2 \mathrm{hr})$ of colony attendance in the context of a circadian rhythm. However, shorter frequencies were also detected (Fig. 5). We believe that such frequencies reflect foraging trips, possibly directed to the same foraging areas. Given the 
relatively stable location of local foraging hotspots-cold Arctic waters, frontal zones and marginal sea ice zone(Jakubas et al. 2013; Jakubas et al. 2014), it is likely that birds often visit the same foraging areas and spend similar lengths of time foraging, especially during bouts of repetitive short trips.

In conclusion, our study indicates that under the conditions of Arctic midnight sun, little auks attend their breeding colony following a regular rhythm, with the highest number of birds in the colony recorded during hours of relatively low sun elevation. However, at the individual level, birds displayed periodicity of $23.2 \mathrm{hr}$ and rather inconsistent daily rhythms. Our study provides evidence for entrainment to subtle light cues under the midnight sun conditions as well as flexibility in little auks' daily activity rhythms, driven by individual time and energy budgets.

\section{Acknowledgements}

The authors thank Lech Iliszko, Gosia Jakimiak, Ania Kośmicka and Magda Hadwiczak for their help in the field and Lech Stempniewicz for his inspirational discussion on the study idea. They also thank George Day for his great help in proofreading of the manuscript and two anonymous reviewers for their helpful and inspirational comments on the manuscript.

\section{Disclosure statement}

The authors report no potential conflict of interest.

\section{Funding}

This study was supported by Norway through the Norwegian Financial Mechanism (ALKEKONGE, grant no. PNRF-234-AI-1/07) and the National Science Centre, Poland (grant no. 2017/25/B/NZ8/01417 to KWJ).

\section{References}

Agostinelli C. \& Lund U. 2017. R package "circular": circular statistics (version 0.4-93). Accessed on the internet at https://r-forge.r-project.org/projects/circular on 19 January 2018.

Ashley N.T., Ubuka T., Schwabl I., Goymann W., Salli B.M., Bentley G.E. \& Buck C.L. 2014. Revealing a circadian clock in captive Arctic-breeding songbirds, Lapland longspurs (Calcarius lapponicus), under constant illumination. Journal of Biological Rhythms 29, 456-469, doi: 10.101177/0748730414552323.
Berge J., Cottier F., Varpe Ø., Renaud P., Falk-Peternsen S., Kwasniewski S., Griffiths C., Søreide J.E., Johnsen G., Aubert A., Bjærke O., Hovinen J., Jung-Madsen S., Tveit M. \& Majaneva S. 2014. Arctic complexity: a case study on diel vertical migration of zooplankton. Journal of Plankton Research 36, 1279-1297, doi: 10.101093/plankt/fbu059.

Błachowiak-Samołyk K., Kwaśniewski S., Richardson K., Dmoch K., Hansen E., Haakon H., Falk-Petersen F. \& Mouritsen L.T. 2006. Arctic zooplankton do not perform diel vertical migration (DVM) during periods of midnight sun. Marine Ecology Progress Series 308, 101-116, doi: 10.103354/meps308101.

Bulla M., Valcu M., Dokter A.M., Dondua A.G., Kosztolányi A., Rutten A., Helm B., Sandercock B.K., Casler B., Ens B.J., Spiegel C.S., Hassell C.J., Küpper C., Minton C., Burgas D., Lank D.B., Payer D.C., Loktionov E.Y., Nol E., Kwon E., Smith F., River Gates H., Vitnerová H., Prüter H., Johnson J.A., St Clair J.J.H., Lamarre J.-F., Rausch J., Reneerkens J., Conklin J.R., Burger J., Liebezeit J., Bêty J., Coleman J.T., Figuerola J., Hooijmeijer J.C.E.W., Alves J.A., Smith J.A.M., Weidinger K., Koivula K., Gosbell K., Niles L., Koloski, L., McKinnon L., Praus L., Klaassen M., Giroux M.-A., Sládeček M., Boldenow M.L., Exo M., Goldtein M.I., Šálek M., Senner N., Rönkä N., Lecomte N., Gilg O., Vincze O., Johnson, O.W., Smith P.A., Woodard P.F., Tomkovich P.S., Battley P., Bentzen R., Lanctot R.B., Porter R., Saalfeld S.T., Freeman S., Brown S.C., Yezerinac S., Székely T., Piersma T., Montalvo T., Loverti V., Pakanen V.-M., Tijsen W. \& Kempenaers B. 2016. Unexpected diversity in socially synchronized rhythms of shorebirds. Nature 540, 109111, doi: 10.101038/nature20563.

Bulla M., Valcu M., Rutten A.L. \& Kempenaers B. 2014. Biparental incubation patterns in a High-Arctic breeding shorebird: how do pairs divide their duties? Behavioural Ecology 25, 152-164, doi: 10.101093/beheco/art098.

Falk K., Egevang C. \& Kampp K. 2000. Measurements of diving depth in dovekies (Alle alle). The Auk 117, 522-525, doi: 10.101642/0004-8038(2000)117.

Favreau A., Richard-Yris M.-A., Bertin A., Houdelier C.T. \& Lumineau S. 2009. Social influences on circadian behavioural rhythms in vertebrates. Animal Behavior 77, 983-989, doi: 10.101016/j.anbehav.2009.01.004.

Gliwicz M.Z. 1986. Predation and the evolution of vertical migration in zooplankton. Nature 320, 746-748, doi: 10.1038/320746a0.

Hansson L., Becares E., Ferna M., Kairesalo T., Miracle M.R., Romo S., Stephen D., Vakkilainen K., Van de Bund W., Van Donk E., Balayla D. \& Moss B. 2007. Relaxed circadian rhythm in zooplankton along a latitudinal gradient. Oikos 116, 585-592, doi: 10.101111/j.2007.0030-1299.15754.x.

Hau M. \& Gwinner E. 1996. Food as a circadian Zeitgeber for house sparrows: the effect of different food access durations. Journal of Biological Rhythms 11, 196-207, doi: 10.1177/074873049601100302.

Huffeldt N.P. \& Merkel F.R. 2016. Sex-specific, inverted rhythms of breeding-site attendance in an Arctic seabird. 
Biology Letters 12, article no. 20160289, doi: 10.101098/ rsbl.2016.0289.

Isaksen K. 1995. The breeding population of little auk (Alle alle) in colonies in Hornsund and northwestern Spitsbergen. In K. Isaksen \& V. Bakken (eds.): Seabird populations in the northern Barents Sea-source data for the impact assessment of the effects of oil drilling activity. Meddelelser 135. Pp. 49-58. Oslo: Norwegian Polar Institute.

Jakubas D., Trudnowska E., Wojczulanis-Jakubas K., Iliszko L., Kidawa D., Darecki M., Błachowiak-Samołyk K. \& Stempniewicz L. 2013. Foraging closer to the colony leads to faster growth in little auks. Marine Ecology Progress Series 489, 263-278, doi: 10.103354/meps 10414.

Jakubas D. \& Wojczulanis-Jakubas K. 2010. Glaucous gull predation on dovekies three new hunting methods. Arctic 63, 468-470, doi: 10.1014430/arctic3335.

Jakubas D., Wojczulanis-Jakubas K., Iliszko L., Darecki M. \& Stempniewicz L. 2014. Foraging strategy of the little auk Alle alle throughout breeding season-switch from unimodal to bimodal pattern. Journal of Avian Biology 45, 551-560, doi: 10.101111/jav.00303.

Keslinka L.K., Wojczulanis-Jakubas K., Jakubas D. \& Neubauer G. 2019. Determinants of the little auk (Alle alle) breeding colony location and size in W and NW coast of Spitsbergen. PLoS One 14, article no. e0212668, doi: 10.101371/journal. pone.0212668.

Kumar V., Singh S., Misra M. \& Malik S. 2001. Effects of duration and time of food availability on photoperiodic responses in the migratory male blackheaded bunting (Emberiza melanocephala). The Journal of Experimental Biology 204, 2843-2848.

Kwasniewski S., Gluchowska M., Jakubas D., Wojczulanis-Jakubas K., Walkusz W., Karnovsky N., Blachowiak-Samolyk K., Cisek M. \& Stempniewicz L. 2010. The impact of different hydrographic conditions and zooplankton communities on provisioning little auks along the west coast of Spitsbergen. Progress in Oceanography 87, 72-82, doi: 10.101016/j.pocean.2010.06.004.

Moe B., Stempniewicz L., Jakubas D. Angelier F., Chastel O., Dinessen F., Gabrielsen G.W., Hanssen F., Karnovsky N.J., Ronning B., Welcker J., Wojczulanis-Jakubas K. \& Bech C. 2009. Climate change and phenological responses of two seabird species breeding in the High-Arctic. Marine Ecology Progress Series 393, 235-246, doi: 10.3354/ meps08222.

Pewsey A., Neuhauser M. \& Graeme D.R. 2014. Circular statistics in R. Oxford: Oxford University Press.

Pietz P.J. 1986. Daily activity patterns of South Polar and brown skuas near Palmer Station, Antarctica. The Auk 103, 726-736, doi: 10.1093/auk/103.4.726.

Steiger S.S., Valcu M., Spoelstra K., Helm B., Wikelski M. \& Kempenaers B. 2013. When the sun never sets: diverse activity rhythms under continuous daylight in free-living Arctic-breeding birds. Proceedings of the Royal Society B 280, article no. 20131016, doi: 10.101098/rspb.2013.1016.

Stempniewicz L. 1986. Factors causing changes in the rhythm of attendance of the little auk Plautus alle (L.) at a colony during the breeding season in Svalbard. Polish Journal of Ecology 34, 247-263.

Stempniewicz L. 1995. Predator-prey interactions between glaucous gull Larus hyperboreus and little auk Alle alle in Spitsbergen. Acta Ornithologica 29, 155-170.

Stempniewicz L. 2001. Little auk (Alle alle). BWP update. The Journal of the Birds of the Western Palearctic 3, 175-201.

Terras A. 1999. Fourier analysis on finite groups and applications. Cambridge: Cambridge University Press.

van Oort B.E.H., Tyler N.J.C., Gerkema Menno P., Folkow L. \& Stokkan K.-A. 2007. Where clocks are redundant: weak circadian mechanisms in reindeer living under polar photic conditions. Naturwissenschaften 94, 183-194, doi: 10.101007/s00114-006-0174-2.

van Oort B.E.H., Tyler N.J.C., Reierth E. \& Stokkan K.-A. 2000. Biological rhythms in Arctic vertebrates. Rangifer 20, 99-112, doi: 10.107557/2.20.2-3.1506.

Wallace M.I., Cottier F.R., Berge J., Tarling G.A., Griffiths C. \& Brierley A.S. 2010. Comparison of zooplankton vertical migration in an ice-free and a seasonally ice-covered Arctic fjord: an insight into the influence of sea ice cover on zooplankton behavior. Limnological Oceanography 55, 831-845, doi: 10.104319/lo.2010.55.2.0831.

Welcker J., Harding A.M.A., Karnovsky N.J., Steen H., Strøm H. \& Gabrielsen G.W. 2009. Flexibility in the bimodal foraging strategy of a High Arctic alcid, the little auk Alle alle. Journal of Avian Biology 40, 388-399, doi: 10.101111/j.1600-048X.2008.04620.x.

Welcker J., Steen H., Harding A.M.A. \& Gabrielsen G.W. 2009. Sex-specific provisioning behaviour in a monomorphic seabird with a bimodal foraging strategy. Ibis 151, 502-513, doi: 10.101111/j.1474-919X.2009.00931.x.

Williams C.T., Barnes B.M. \& Buck C.L. 2015. Persistence, entrainment, and function of circadian rhythms in polar vertebrates. Physiology 30, 86-96, doi: 10.101152/physiol.00045.2014.

Wilson R., Culik B., Coria N.R., Adelung D. \& Spairani H. 1989. Foraging rhythms in Adelie penguins (Pygoscelis adeliae) at Hope Bay, Antarctica; determination and control. Polar Biology 10, 161-165, doi: 10.1007/BF00238490.

Wojczulanis-Jakubas K., Araya-Salas M. \& Jakubas D. 2018. Seabird parents provision their chick in a coordinated manner. PLoS One 13, e0189969, doi: 10.101371/journal. pone.0189969.

Wojczulanis-Jakubas K., Jakubas D. \& Stempniewicz L. 2005. Changes in the glaucous gull predatory pressure on little auks in southwest Spitsbergen. Waterbirds 28, 430-435, doi: 10.101675/1524-4695(2005)28.

Wojczulanis-Jakubas K., Jakubas D., Walkusz W. \& Wennerberg L. 2006. Differences in food delivered to chicks by males and females of little auks (Alle alle) on south Spitsbergen. Journal of Ornithology 147, 543-548, doi: 10.101007/s10336-006-0077-1.

Wood S.N. 2011. Fast stable restricted maximum likelihood and marginal likelihood estimation of semiparametric generalized linear models. Journal of the Royal Statistical Society $B$ 73, 3-36, doi: 10.101111/j.1467-9868.2010.00749.x. 